\title{
Transverse priority phase sensitive optical coherence tomography
}

Jeff Fingler, Jon Williams, Zahid Yaqoob, Changhuei Yang, Richard Haskell, et al.

Jeff Fingler, Jon Williams, Zahid Yaqoob, Changhuei Yang, Richard Haskell, Scott Fraser, "Transverse priority phase sensitive optical coherence tomography," Proc. SPIE 6079, Coherence Domain Optical Methods and Optical Coherence Tomography in Biomedicine X, 607922 (20 February 2006); doi: 10.1117/12.648997

SPIE. Event: SPIE BiOS, 2006, San Jose, California, United States 


\title{
Transverse Priority Phase Sensitive Optical Coherence Tomography
}

\author{
Jeff Fingler $^{\mathrm{a}}$, Jon Williams ${ }^{\mathrm{b}}$, Zahid Yaqoob ${ }^{\mathrm{c}}$, Changhuei Yang ${ }^{\mathrm{c}}$, Richard Haskell ${ }^{\mathrm{d}}$, Scott Fraser ${ }^{\mathrm{b}}$ \\ Departments of Applied Physics ${ }^{\mathrm{a}}$, Biology ${ }^{\mathbf{b}}$ and Electrical Engineering ${ }^{\mathbf{c}}$, \\ California Institute of Technology, Pasadena, CA 91125 \\ ${ }^{\mathbf{d}}$ Harvey Mudd College, Claremont, CA
}

\begin{abstract}
A variation on the standard time domain optical coherence tomography (TDOCT) system is presented. Using an inexpensive piezoelectric stack to modulate the reference mirror position, the amplitude and phase of the sample reflection is determined without scanning. With the primary scan in the transverse direction, en face and B-scan OCT images can be readily produced with phase information. This project plans to use the dynamic phase information to add an extra level of contrast to the images, based on the motion of the scatterers.
\end{abstract}

Keywords: optical coherence tomography, en face, transverse priority, phase sensitive, phase contrast, motion sigma

\section{INTRODUCTION}

Time domain optical coherence tomography (TDOCT) traditionally scans the reference arm to produce interference fringes and perform a single depth scan. In several cases, en face (transversal) images are the preferred form to view the data. In order to produce en face images when the depth scan is the primary direction, the data must be extracted from a $3 \mathrm{D}$ data set. In order to produce a heterodyne signal to allow a direct measure of an en face OCT, a phase modulation is used to sample the fringe information. While several groups have used scanner alignment ${ }^{[1]}$, acousto-optic modulators ${ }^{[2]}$, and full field imaging ${ }^{[3]}$ to produce en face images, this system uses a piezoelectric stack to modulate the reference arm for each pixel in the image. By controlling the amplitude of the reference arm oscillation ${ }^{[4,5]}$ phase information can be extracted from the OCT signal, independent of the primary scan direction.

By oscillating the phase of the reference arm (see Figure 1) sinusoidally with a displacement amplitude $d_{o}$ and frequency $\omega$, the interference signal measured at the photodiode is given by:

$$
V_{\text {interference }}=V_{0} \cos [\alpha \sin \omega t+\phi]
$$

where $\alpha=4 \pi d_{0} / \lambda$ and $\phi$ is the phase difference between the sample and reference arms. This signal can be expanded:

$$
\begin{gathered}
V_{\text {interference }}=V_{0}\left[J_{0}(\alpha)+2 J_{2}(\alpha) \cos 2 \omega t+2 J_{4}(\alpha) \cos 4 \omega t+\ldots\right] \cos \phi \\
-V_{0}\left[2 J_{1}(\alpha) \cos \omega t+2 J_{3}(\alpha) \cos 3 \omega t+\ldots\right] \sin \phi
\end{gathered}
$$

This expansion continues for higher harmonics with even Bessel functions multiplied by $\cos \phi$ and odd Bessel functions multiplied by $\sin \phi$. The $2 \omega$ and $3 \omega$ components of the power spectrum of the interference signal were chosen for intensity and phase calculations. By constraining the amplitude of the phase modulation to $\mathrm{d}_{\mathrm{o}}=0.300 \lambda$ such that $J_{2}(\alpha)^{2}=J_{3}(\alpha)^{2}=0.30$, the intensity and phase are calculated using the $2 \omega$ and $3 \omega$ components of the power spectrum:

\footnotetext{
Coherence Domain Optical Methods and Optical Coherence Tomography in Biomedicine $X$

edited by Valery V. Tuchin, Joseph A. Izatt, James G. Fujimoto, Proc. of SPIE Vol. 6079

607922, (2006) - 1605-7422/06/\$15 - doi: 10.1117/12.648997
}

Proc. of SPIE Vol. $6079607922-1$ 


$$
\begin{aligned}
& P_{2}=0.30 V_{0}^{2} \cos ^{2} \phi \\
& P_{3}=0.30 V_{0}^{2} \sin ^{2} \phi
\end{aligned}
$$

Summing the two harmonics from (3) produces an OCT signal independent of the phase $\phi$. Dividing the two power spectrum terms allows for the phase $\phi$ to be determined. This method of OCT signal extraction allows for transverse priority scanning such that en face (C-scan) as well as cross sectional (B-scan) images can be produced. Faster phase modulation speed is possible, allowing for faster data acquisition and imaging than demonstrated in the presented system.

\section{METHOD}

The current experimental setup, shown in Figure 1, uses a fiber based Michelson interferometer setup with a piezoelectric stack to oscillate the reference arm phase at $\omega=75.5 \mathrm{kHz}$. The minimum transverse and axial resolutions of the OCT system are approximately 20 microns and 7 microns, respectively. A calibrated SNR of $93 \mathrm{~dB}$ for a Fast Fourier Transform power spectrum (FFT) of $100 \mu \mathrm{s}$ (time per pixel) and $91 \mathrm{~dB}$ for $50 \mu \mathrm{s}$ has been achieved. The lateral resolution is determined by the sum of the spot size $(20 \mu \mathrm{m})$ and the scanned distance during the power spectrum measurement. With a scan velocity of approximately $0.14 \mathrm{~m} / \mathrm{s}$, the dynamic lateral resolution of the transverse priority OCT system with a $50 \mu$ sFT is theoretically $27 \mu \mathrm{m}$.

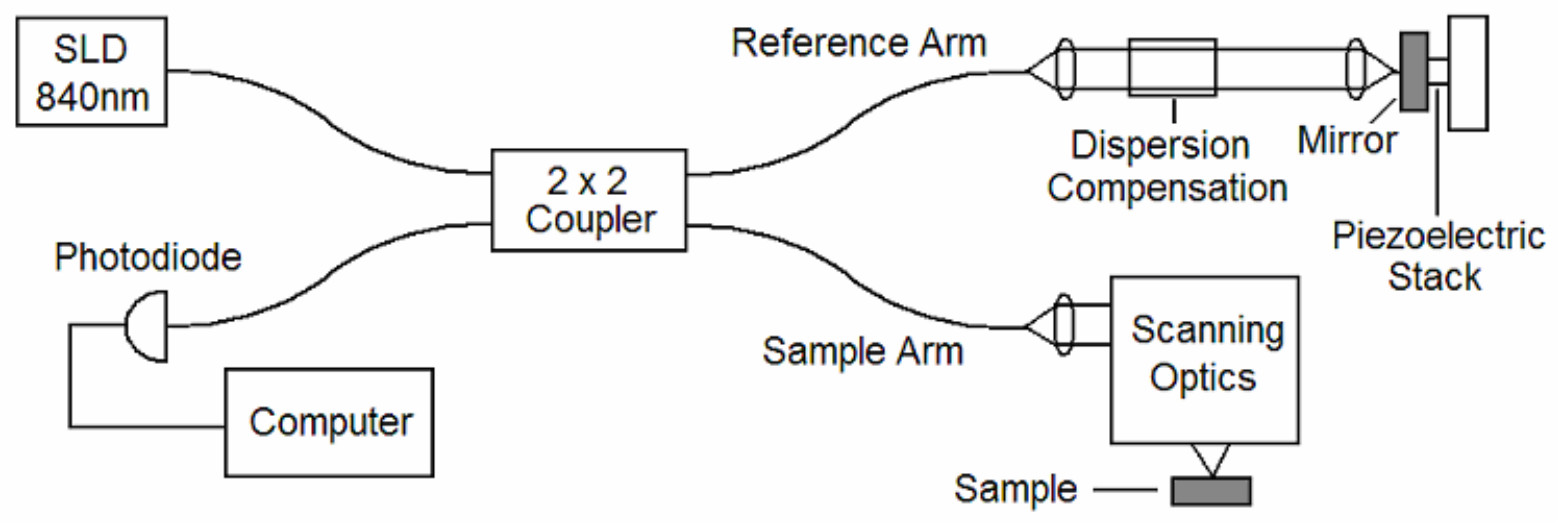

Figure 1 - Schematic of en face phase sensitive OCT system

One of the advantages of the proposed en face OCT system is the flexibility of analysis of the raw data from the photodiode. If the sample has phase variations on the order of $\pi / 2$ within the time of the FFT, standard analysis techniques involving successive FFTs would be unable to view the finer structure of the phase variation. For a finer view of the phase, this system uses a sliding FFT window of size $T$ to look at average phase changes over scales much smaller than T. For example, data acquisition at $1 \mathrm{MHz}$ allows for a 50 data point $(50 \mu \mathrm{s})$ FFT window which can be shifted by single data points to analyze the average phase changes over a $1 \mu$ s time scale. This technique is graphically represented in figure 2 .

High density phase imaging allows visualization of motions on the order of the acquisition speed. Current techniques like spectral domain optical coherence tomography (SDOCT) calculate the average phase of reflectors over successive FFTs corresponding to each line scan of the spectrometer ${ }^{[6]}$ Successive phase measurements are limited by an inability to visualize higher frequency motions. Figure 3 demonstrates measurements of phase motion above the expected maximum resolvable frequency as described by the Nyquist theorem for data acquisition at a rate of $20 \mathrm{kHz}$. The sliding window technique demonstrates that motion at the expected frequency of $16.6 \mathrm{kHz}$ can be observed while successive phase measurements are unable to observe this oscillation. 


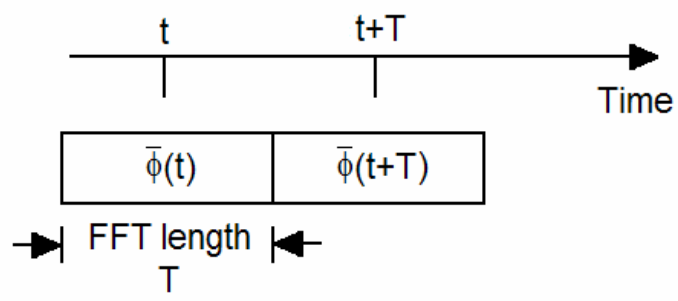

a) Sequential segmentation
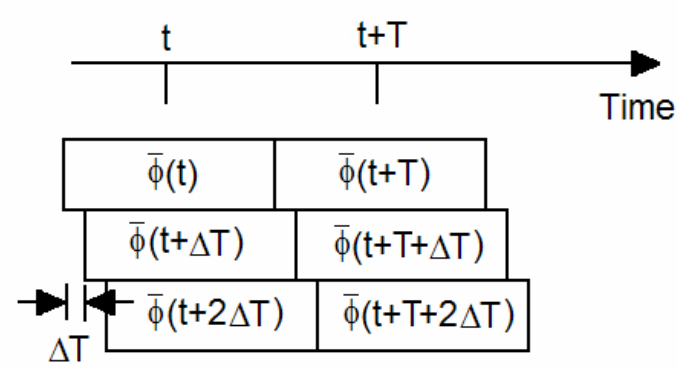

b) Overlapping segmentation with FFT window shift $\Delta T$

Figure 2 - Illustration of segmentation of raw data for FFT analysis in a) standard OCT imaging with successive FFTs and b) high density phase imaging using a sliding FFT technique
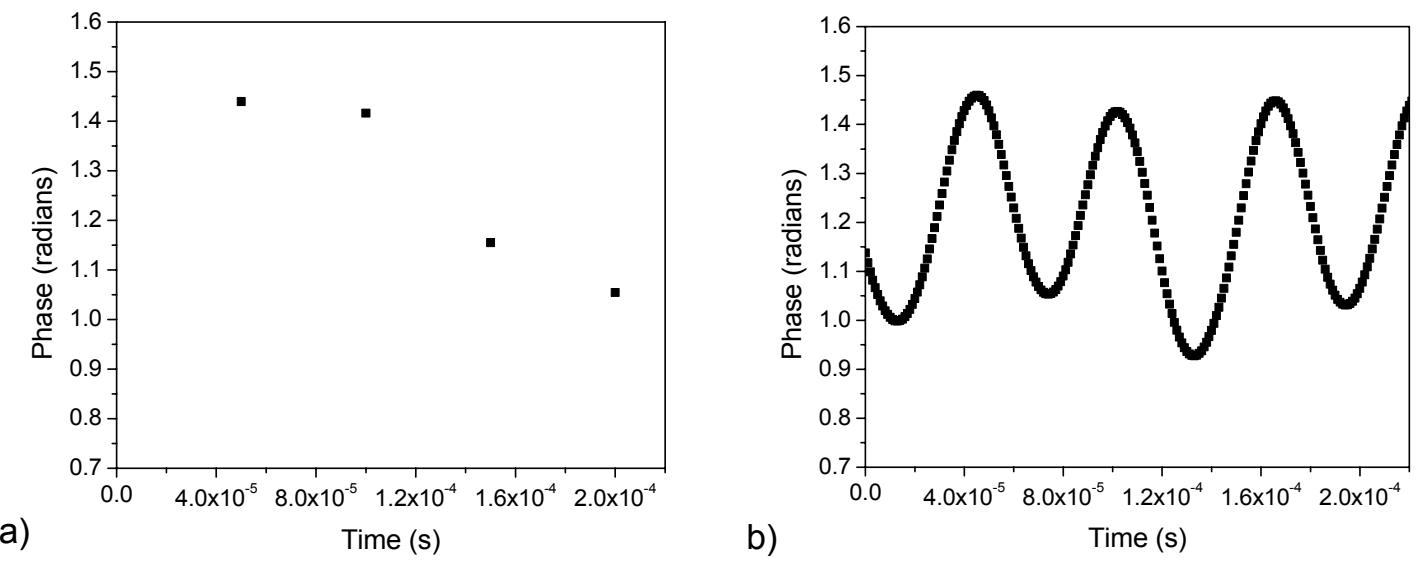

Figure 3 - Visualization of Phase Motion for $20 \mathrm{kHz}$ acquisition rate for $16.6 \mathrm{kHz}$ motion using

a) successive FFT measurements and b) sliding FFT window technique for high density phase imaging (1 $\mu$ sFT shift)

It is noted that the motion observed in Figure $3 \mathrm{~b}$ ) is not true phase motion but the motion of the average phase over the time of the FFT. Further analysis is required to determine the expected discrepancy between actual motion and average motion with this technique. The main purpose of this technique is improved visualization to determine whether motion is occurring at a frequency much higher than can usually be seen for a given acquisition rate. Ultimate limits to the capabilities of this technique have not yet been determined.

An additional capability of this system comes from the fact that the OCT signal is calculated from two separate measurements from the power spectrum of the interference signal. The $2 \omega$ and $3 \omega$ components of the power spectrum each depend differently on the phase of the system as described by equation (3), and the changes in these components over time for phase changes are related to each other as shown in equation (4). Figure 4 demonstrates normalized correlation plots for power spectrum component changes for two cases with similar standard deviations of phase which has one caused by phase motion and another by phase inaccuracy caused by fundamental noise limitations. ${ }^{[7]}$

$$
\begin{gathered}
\Delta P_{2}(t)=-0.60 V_{0}^{2} \cos \phi \sin \phi[\Delta \phi(t)] \\
\Delta P_{3}(t)=0.60 V_{0}^{2} \cos \phi \sin \phi[\Delta \phi(t)]=-\Delta P_{2}(t)
\end{gathered}
$$


The normalization of the power spectrum component changes used for the correlation plots in figure 4 are with respect to the interference amplitude, of the form $\Delta P_{2}(t) /\left(P_{2}+P_{3}\right)$ against $\Delta P_{3}(t) /\left(P_{2}+P_{3}\right)$. A negative correlation corresponds to phase changes while phase accuracy limited measurements do not have this form. Both cases chosen for figure 4 have the same standard deviation (motion sigma) of phase but have different causes.
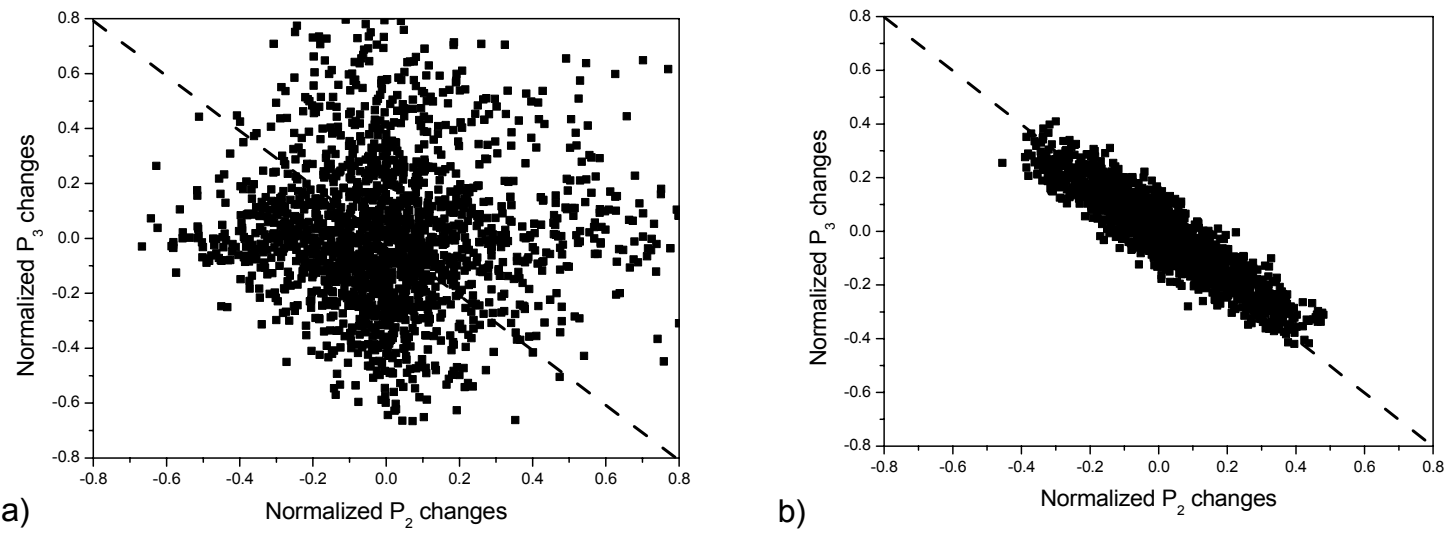

Figure 4 - Correlation plots of normalized changes in power spectrum components for cases with motion sigma of 0.22 radians:

a) phase accuracy limited measurements at $50 \mu \mathrm{s}$ and b) system motion noise limited for $2 \mathrm{~ms}$

Dashed line corresponds to expected form for negative correlation.

Figure 4 a) demonstrates a reflector with a $\mathrm{SNR}=16$ for a short time period of $50 \mu$ s where the phase error due to the low OCT signal dominates the phase noise in the system. Because the system noise is not dominated by motion, the correlation plot appears random and does not follow along the dotted line corresponding to negative correlation. The plot in figure $4 \mathrm{~b}$ ) demonstrates a reflector with $\mathrm{SNR}=600$ at a time separation of $2 \mathrm{~ms}$, where system motion noise dominates but is not the only noise source. The phase error due to the SNR of the data explains the deviation from a perfect correlation.

\section{EXPERIMENTAL RESULTS}

\subsection{System Noise}

The phase measured by the system is the relative phase between the sample arm and the reference arm. In order to use phase motion of scatterers within a sample as a contrast mechanism, the motion noise of the system must be fully characterized. There have been very few published results containing phase noise of OCT systems, but the ones which exist $^{[8],[9]}$ generally only deal with the case of successive phase measurements (approximately $30 \mu$ s separation of measurements). These results are usually presented as a minimum detectable flow velocity for Doppler OCT measurements.

The phase noise in this system is presented through the motion sigma, or the standard deviation of the phase changes for a series of phase measurements. The motion sigma was plotted as a function of the time separation of the phase changes used in the calculation. The system has gone through several variations and each time the system noise has been characterized. Figure 5 demonstrates the phase noise measured in four different cases with a mirror as the sample: a) a galvanometer scanner (Cambridge Technologies $6210 \mathrm{H}$ scanners) in the system in operational mode but with a static position voltage applied, b) a galvanometer scanner in the system with no power going to the scanners at all, and c) a two dimensional piezo-actuated mirror scanner (Physik Instrumente S-334 Tip Tilt Mirror) in the system in operational mode with a static position voltage applied. While the amplitude of the phase noise demonstrated in Figure 5 a) for an operational galvanometric scanner depends on the alignment of the system, there was always observed a significant difference in noise measured depending on whether operational power was supplied to the galvanometric scanner controller. 


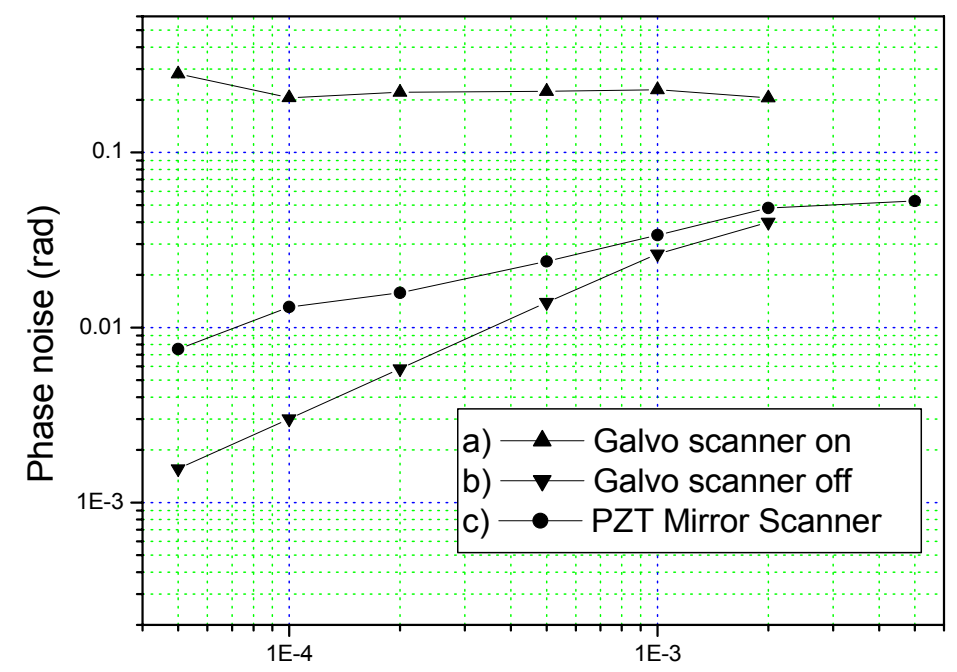

Time separation of phase measurements (s)

Figure 5 - System phase noise as a function of time for a) galvo scanner on b) galvo scanner off, c) piezo-actuated 2D mirror scanner on

\subsection{Phase Measurement Limitations}

Several theory ${ }^{[7]}$ and experimental ${ }^{[9]}$ papers have demonstrated a relationship between the phase measurement accuracy of a reflector and the OCT signal strength of that reflector. The phase inaccuracy and the system noise are both phase noise sources which contribute to the limiting factors of phase noise measurement. In order to demonstrate which noise source is dominant in different regimes, an M-Scan was completed on a stationary mirror. For each depth point in the scan, the standard deviation of the phase was calculated for time separations of $50 \mu \mathrm{s}$ up to $15 \mathrm{~ms}$. For three different time separations, the phase noise was plotted as a function of the OCT signal.

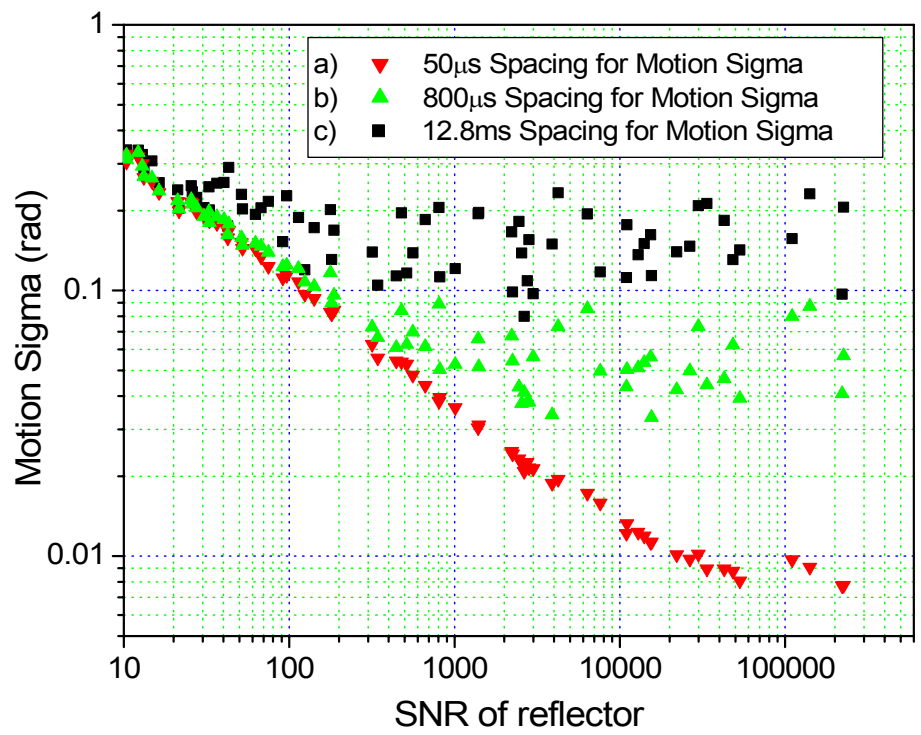

Figure 6 - System phase noise as a function of OCT signal for a) $50 \mu \mathrm{s}$, b) $800 \mu \mathrm{s}$ and c) $12.8 \mathrm{~ms}$ 
Figure 6 demonstrates the different time and intensity regimes where each noise source dominates. System motion noise dominates the calculated phase noise in cases with very strong OCT signal and also in cases with long time spacing between phase measurements. Signal limited measurements, which has a definite relation to the OCT signal, is most strongly dependant in the case of short time spacing which is the most common technique of phase measurement in OCT systems.

\subsection{Phase Contrast Work}

In order to determine the phase capabilities of the system in a test case with OCT signals comparable to biological systems with known motion, the signals from polystyrene microspheres (Polysciences, Inc.) were analyzed. Suspensions of polystyrene microspheres in water were imaged using the phase sensitive system. Data was taken for each scatterer using a static position for all three scanning directions for approximately $100 \mathrm{~ms}$. The motion sigma calculations were fit to equation (5), which is the quadratic combination of the two dominant noise factors contributing to phase calculations. The noise due to SNR-limited phase inaccuracy is described by A, a term which is independent of the time separation of phase measurements. The noise due to motion of the scatterers follows the form of $D T^{\gamma}$ for a time separation $\mathrm{T}$ for phase measurements. The expected value for Brownian motion of particles is $\gamma=1 / 2$.

$$
\sigma_{\phi}(T)=\sqrt{A^{2}+\left(D T^{\gamma}\right)^{2}}
$$

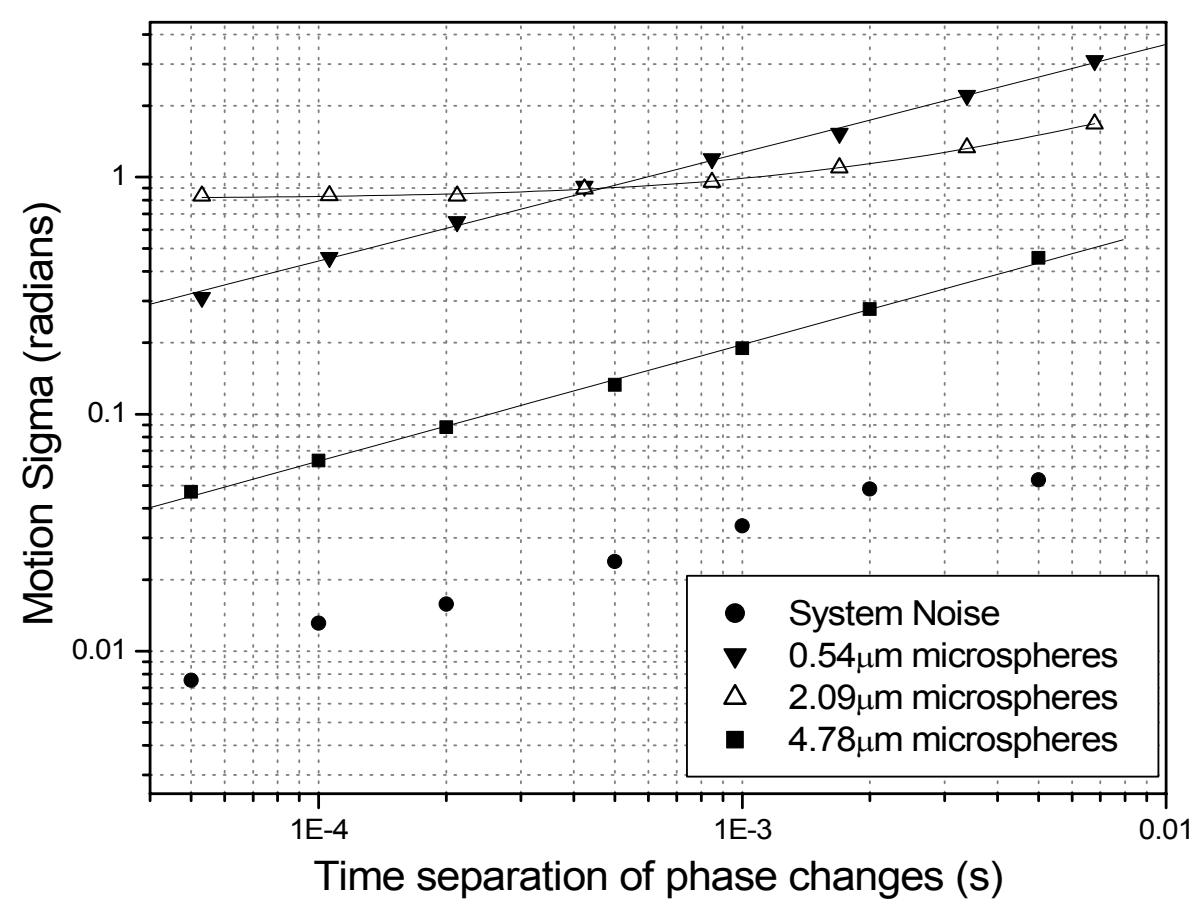

Figure 7 - Phase variation measurements for OCT signals of polystyrene microspheres in water. Data shown is for $0.54 \mu \mathrm{m}, 2.09 \mu \mathrm{m}$ and $4.78 \mu \mathrm{m}$ diameter spheres. System noise is included for comparison.

Microsphere data is fit to equation (5) with the assumption of Brownian motion.

The phase motion is linearly related to the position motion of the sample. The motion sigma of the phase is proportional to the RMS fluctuations of position of the sample:

$$
\sqrt{\left\langle\Delta x^{2}\right\rangle}=\sigma_{x}(T)=\frac{\lambda}{4 \pi} \sigma_{\phi}(T)
$$


From figure 7 , it can be easily seen that the data of the $0.54 \mu \mathrm{m}$ and $4.78 \mu \mathrm{m}$ diameter microspheres fits to equation (5) in the case where $\mathrm{A}$ is approximately equal to zero. For these data sets, the OCT signal was high enough that even at $50 \mu \mathrm{s}$, the motion of the scatterers was greater than the error in phase calculation. The $2.09 \mu \mathrm{m}$ diameter spheres demonstrate a case where the OCT signal is not ideal and phase inaccuracy dominates the motion sigma calculation for small time separations. For this data, only a fit to equation (5) can truly determine the motion of the scatterer. Also, trying to determine contrast based on the motion sigma at one time point may cause inaccuracies. Due to the low OCT signal for the $2.09 \mu \mathrm{m}$ spheres, the motion sigma data crosses the $0.54 \mu \mathrm{m}$ sphere data which has higher OCT signal but larger scatterer motion.

There is the possibility that motion contrast can arise from similar amplitude motion but different temporal signatures. Looking at the case of oscillatory motion, the motion sigma can be calculated in the same fashion as before but the results do not fit to equation (5). To determine the temporal form of the motion sigma of oscillatory phase changes, a mirror mounted on a piezoelectric crystal was placed in the sample arm of the system. While measuring phase changes with an FFT window of $53 \mu \mathrm{s}$, the sample mirror was oscillated at $910 \mathrm{~Hz}$. Motion sigma calculations for this motion are plotted in Figure 8, along with the theoretical expected motion sigma for oscillatory motion of frequency $910 \mathrm{~Hz}$ and amplitude of 0.13 radians.

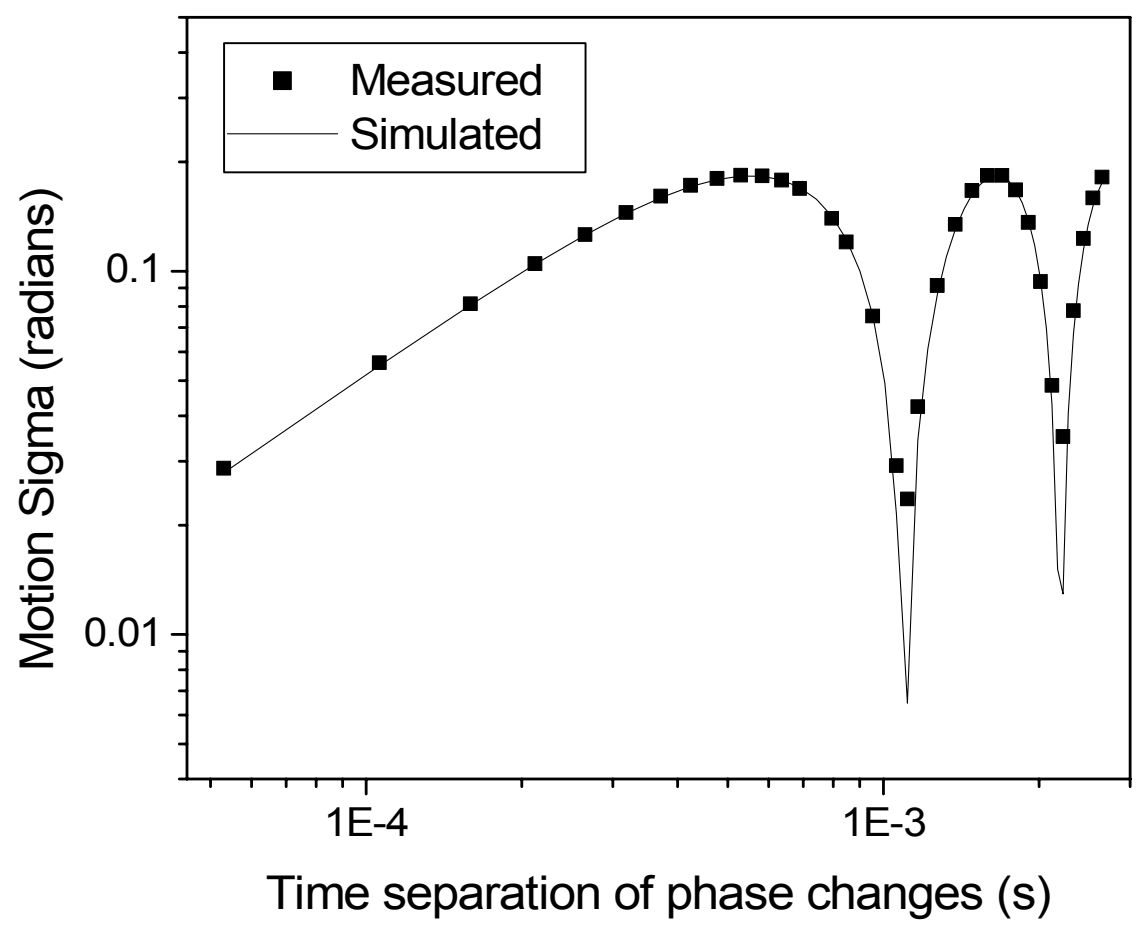

Figure 8 - Phase noise measured from $910 \mathrm{~Hz}$ Piezo oscillated sample mirror. Simulated motion sigma for $910 \mathrm{~Hz}$ sinusoidal oscillation of amplitude 0.13 radians overlaid over data.

Figures 7 and 8 demonstrate how different types of motion (oscillatory versus random) produce different temporal signatures in motion sigma calculations. In most cases it is not expected that a purely sinusoidal oscillation will occur with a sample motion, therefore any dips observed in the motion sigma for oscillatory motion as seen in figure 8 will probably not be as significant. If oscillatory motion is combined with another motion or a low OCT signal, the resulting motion sigma is the quadratic sum of these components and will reduce or remove these dips from the calculations. Further analysis is required to determine the full extent of the motion contrast capabilities using this type of data. 


\subsection{Phase Contrast Image}

To demonstrate the imaging capabilities of phase contrast, a B-scan OCT image was taken of a capillary tube oriented transversely to the imaging direction with no flow occurring within the tube. The phase contrast image was created by using the motion sigma data for each pixel after removing the contribution due to SNR-limited phase inaccuracy. The total dwell time per pixel for the phase contrast image was 10ms. The contrast image contains intensity threshold for the OCT image of SNR $>1.25$ to remove the data pixels created by pure noise.

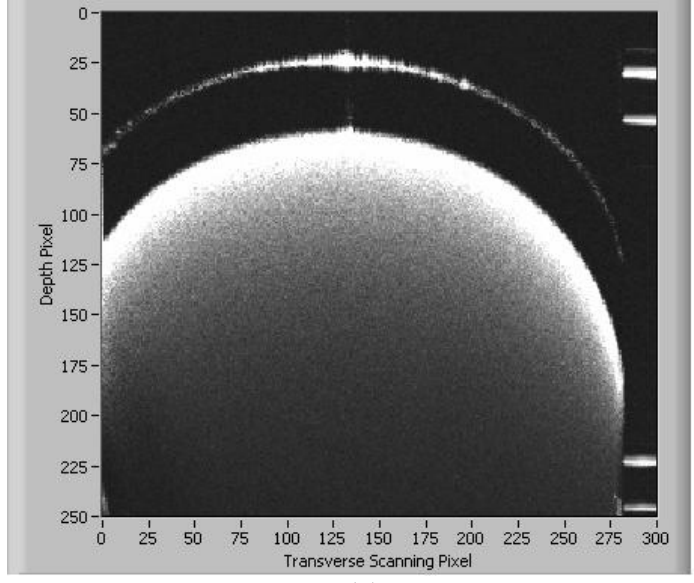

(a)
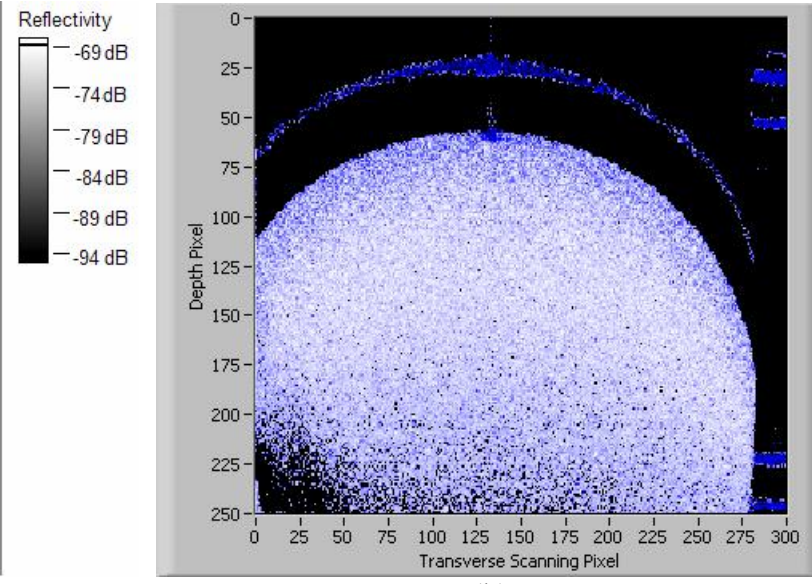

(b)

Figure 9 - (a) OCT Intensity and (b) Phase contrast images for capillary tube containing 20\% intralipid without flow. Depth of image is $2 \mathrm{~mm}$.

In Figure 9, it can be seen that the phase contrast due to the reflections of the top of the capillary tube and the multiple reflections on the right side of the image do not have a zero value. As seen in Figures 5 and 7, the motion noise of this system is not constant over time and determines a lower limit on the possible contrast with this system. The dynamic range of the phase contrast possible with this system is approximately $10 \mathrm{~dB}$. It should also be noted that the phase contrast calculated for Figure 9 (b) remains approximately constant over the depth of the intralipid even until the SNR of the OCT signal goes to 1 and the threshold has taken effect. If motion sigma was imaged in this situation, the value would continue increasing with depth due to increasing phase inaccuracy due to decreasing OCT signal.

Preliminary results in this system have demonstrated contrast in a capillary tube oriented transversely to the imaging direction with no flow. It has also been demonstrated that the motion sigma temporal curves have forms which depend on the type of motion in the sample, which may be used as a different type of contrast beyond the amplitude of phase motion demonstrated here. Future work plans to use these techniques to differentiate samples with dissimilar phase variations.

\section{REFERENCES}

[1] A. Gh. Podoleanu, G. M. Dobre, D. A. Jackson, Optics Letters 23 (3), 147-149, (1998)

[2] C.K. Hitzenberger, P. Trost, P.W. Lo, Q. Zhou, Optics Express 11 (21), 2753-2761 (2003)

[3] A. Dubois, K. Grieve, G. Moneron, R. Lecaque, L. Vabre, C. Boccara, Applied Optics, 43 (14), 2874-2883, (2004)

[4] B.M. Hoeling, A.D. Fernandez, R.C. Haskell, S.E. Fraser et al, Optics Express 6 (7), 136-146, (2000)

[5] B.M. Hoeling, A.D Fernandez, R.C. Haskell, D.C. Petersen, Rev. Sci. Instruments 72 (3), 1630-1633 (2001)

[6] R.A. Leitgeb, L. Schmetterer, C.K. Hitzenberger et al, Optics Letters 29 (2), 171-173, (2004)

[7] S. Yazdanfar, C.H. Yang, M.V. Sarunic, J.A. Izatt, Optics Express 13 (2), 410-416, (2005)

[8] B.R. White, M.C. Pierce, N. Nassif et al, Optics Express 11 (25), 3490-3497, (2003)

[9] B.J. Vakoc, S.H. Yun, J.F. de Boer et al, Optics Express 12 (14), 5483-5493, (2005) 